\title{
Correlación entre el clima educacional y el síndrome de desgaste profesional en los programas de residencia de un hospital universitario
}

\author{
Correlation between the educational environment and burn- \\ out syndrome in residency programs at a university hospital
}

\author{
Dr. Julián Llera ${ }^{a}$ y Dr. Eduardo Durante ${ }^{b}$
}

\section{RESUMEN}

Introducción. El clima educacional (CE) se relaciona con los logros y la satisfacción. Se ha comunicado una alta prevalencia del síndrome de desgaste profesional (BO) en la residencia. El objetivo de este trabajo fue evaluar la correlación entre el CE y la presencia de BO en los programas de residencia de un hospital universitario.

Población y métodos. Se evaluaron residentes de cinco programas del Hospital Italiano de Buenos Aires.Se registró: especialidad, año de residencia, sexo, nacionalidad y convivencia.

El CE se midió con el Postgraduate Hospital Education Environment Measure, PHEEM, que evalúa autonomía, soporte y enseñanza. El BO se midió con el Maslach Burnout Inventory (MBI) que evalúa agotamiento, despersonalización y realización personal.

Resultados. Participaron 92 residentes. La media de CE total fue de 106,8 (clima más positivo que negativo). El 19,6\% de los evaluados mostraron BO. Hubo diferencias significativas de CE entre las poblaciones con y $\sin \mathrm{BO}$; medias: 98,7 contra $108,8(p=0,0056)$.

Se observó una correlación significativa negativa entre CE/agotamiento $(r=-0,24)$ y CE/ despersonalización $(\mathrm{r}=-0,35)$ y una correlación significativa positiva entre CE y realización $(r=0,44)$. Mayor correlación en igual sentido se observó entre la subescala de autonomía del PHEEM y el BO ( $r=-0,58 ;-0,41$ y 0,46 , respectivamente).

a. Servicio de Clínica Pediátrica, Departamento de Pediatría.

b. Servicio de Medicina Familiar y Comunitaria.

Hospital Italiano de Buenos Aires.

\section{Correspondencia:}

Dr. Julián Llera: julian. llera@hospitalitaliano. org.ar

Conflicto de intereses: Ninguno que declarar.

Recibido: 13-5-2013

Aceptado: 12-8-2013 el contexto y se relaciona con factores vinculados al trabajo con otros miembros del equipo de salud y con situaciones que tienen que ver con la cultura de las instituciones.

Genn y Harden ${ }^{1-3}$ destacan la extraordinaria complejidad del ambiente en la educación médica. Subrayan que si se quiere describir o evaluar el currículo de una escuela de medicina es necesario considerar el ambiente organizacional que define el CE. Esta evaluación permite planificar modificaciones curriculares, con una mejora continua de la calidad, mecanismo que define a la escuela de medicina como una organización que aprende. ${ }^{4}$

McAleer ${ }^{5}$ enumera los componentes claves del CE (la habilidad de los maestros, los materiales y métodos de enseñanza, los objetivos y la evaluación, hasta la vida social, etc.) y, como refiere Hutchinson ${ }^{6}$ en la teoría de aprendizaje del adulto, tiene tanta relevancia el contexto y el clima como la formación educacional ofrecida y la experiencia compartida. La motivación extrínseca desempeña un papel especial para lograr un aprendizaje significativo.

Por otro lado, son llamativos los múltiples informes que describen una alta prevalencia de indicadores de tensión emocional, tanto entre los estudiantes de medicina como entre los residentes. ${ }^{7-10}$ Este tipo de manifestaciones de la conducta se describe como el síndrome de desgaste profesional o burnout (BO). Según Maslach, el BO tiene tres dimensiones: agotamiento emocional, 
despersonalización y cinismo, y sentimiento de ineficacia. La autora creó el Maslach Burnout Inventory (MBI), ${ }^{11}$ en el cual un puntaje alto en los dominios de agotamiento emocional o despersonalización es indicativo de un síndrome de BO clínicamente significativo. Este instrumento se considera el método de referencia para identificar el $\mathrm{BO} .^{12}$

Después de relevar la bibliografía no se encontraron estudios de la correlación entre el CE y el $\mathrm{BO}$, realizados con instrumentos validados, en el contexto de los programas de residencia.

\section{OBJETIVO}

Evaluar la correlación entre el CE y el BO en los programas de residencia del Hospital Italiano de Buenos Aires.

\section{MATERIAL Y MÉTODOS}

El estudio se realizó en el Hospital Italiano de Buenos Aires en mayo de 2011. Se invitó a participar a todos los programas de residencia del hospital que estuvieran dispuestos a evaluar la totalidad de sus residentes (de todos los años). Se diseñó un estudio de corte transversal, observacional.

Las variables para analizar fueron: sexo, especialidad, año de la residencia (1, 2, 3 o 4), convivencia (solo o acompañado) y nacionalidad (argentino o extranjero). Se evaluó CE y BO.

Para la medición de CE se utilizó el PHEEM. Este instrumento, creado por Roff y cols., ${ }^{13}$ cuenta con 40 ítems operacionalizados con una escala de Likert de 0 a 4 . Evalúa tres dominios: percepción del rol de autonomía, percepción de la enseñanza y percepción del soporte social. La suma total del cuestionario es de 160 puntos. El cuestionario y su interpretación se muestran en el Anexo electrónico 1.

Para la evaluación de BO se utilizó el Maslach Burnout Inventory Human Service Survey, ${ }^{11}$ que cuenta con 22 ítems, con un puntaje en una escala de Likert entre 0 y 6 . El cuestionario y su interpretación se muestran en el Anexo electrónico 2.

El resultado principal fue la correlación entre CE y cada subescala del MBI. Se aceptó la medición del CE como variable continua siguiendo las sugerencias del autor del instrumento.

Se analizó la correlación entre las tres dimensiones del MBI y las tres subescalas del PHEEM.
Se analizaron los datos de evaluación de CE y BO en forma general y según las variables demográficas.

Para el análisis estadístico se dicotomizaron las tres dimensiones del MBI y se definió la presencia del síndrome de BO si el puntaje correspondiente a cada residente era superior a 26 en la subescala de agotamiento emocional (A), superior a 9 en la subescala de despersonalización (D) e inferior a 34 en la subescala de realización personal (R).

Posteriormente, se compararon las medias del resultado global de evaluación del clima entre los residentes con $\mathrm{BO}$ y sin él.

Por otro lado, para evaluar el grado de predicción del clima sobre el $\mathrm{BO}$, se dicotomizó la evaluación del clima educacional por su mediana $(109,4)$. Se evaluó la confiabilidad del PHEEM con el coeficiente alfa de Chronbach.

\section{Tamaño muestral}

En nuestra institución se realizó un estudio piloto, en el año 2010, aplicando ambos instrumentos (el PHEEM y el MBI-Human Services Survey) a los 34 residentes de Pediatría.

Teniendo en cuenta los resultados preliminares, se calculó un tamaño muestral de 95 encuestados, para un coeficiente de correlación (r) esperado de 0,4 (para la subescala de realización, que es la que mostró mayor correlación con la medición del clima en el estudio piloto previo), con un poder de $90 \%$ y un nivel de confianza de $99 \%$, previendo una pérdida de datos del $10 \%$.

\section{Análisis estadístico}

Los datos continuos que presentaron distribución normal se analizaron con la prueba de la $t$ para la diferencia de las medias, Anova y coeficiente de correlación de Pearson. Con los datos categóricos se utilizó la prueba de la $\chi^{2}$ y la prueba exacta de Fisher cuando correspondía. Se tomó como significativo un valor de $p$ menor de 0,05. Los datos fueron analizados con Stata 11.0, Statistic/Data Analysis, Stata Corp.

\section{Aspectos éticos}

A mbos cuestionarios fueron autoadministrados, anónimos y de respuesta voluntaria. Se aseguró la confidencialidad de los datos. Se incorporaron los programas de residencia de la Institución que aceptaron participar en el estudio con la totalidad de sus residentes. Los instrumentos eran entregados y recuperados por los jefes de residentes respectivos 
manteniendo las condiciones de anonimato y entregados posteriormente a los autores para el análisis.

El trabajo fue evaluado y aprobado por el Comité de Ética de Protocolos de Investigación del Hospital Italiano de Buenos Aires.

\section{RESULTADOS}

La Institución cuenta con 15 programas de residencia, con 196 residentes totales. Aceptaron participar 6 programas de residencia (pediatría, clínica, medicina familiar, cardiología y terapia intensiva). Se completaron 92 encuestas (100\% de los residentes de pediatría, medicina familiar y terapia intensiva, $85 \%$ de los residentes de cardiología y $45 \%$ de los residentes de clínica médica). La tasa de respuesta fue del 100\%, tanto para las preguntas del PHEEM como del MBI. La población evaluada se describe en la Tabla 1.

La evaluación del CE arrojó una media de clima total de 106,8 con una desviación estándar de 13,98 (rango 70 a 155). Esta medida de CE expresa un clima más positivo que negativo (véase Anexo 1).

La evaluación de las subescalas del PHEEM se muestra en la Tabla 2.

La única variable demográfica que mostró diferencia en la evaluación del CE fue entre las especialidades (Anova F: 2,96; $p=0,0241$ ) (resultados no mostrados).

Los resultados de la evaluación del $\mathrm{BO}$ se muestran en la Tabla 3. El análisis con Anova de las subescalas de BO solo mostraron diferencias significativas según la especialidad en agotamiento (F: 2,$85 ; p=0,0283) \mathrm{y}$

Tabla 1. Población de residentes encuestados

\begin{tabular}{lcc}
\hline & & Total (\%) \\
\hline Especialidad & Pediatría & $29(31,5)$ \\
& Clínica médica & $20(21,7)$ \\
& Medicina familiar & $14(15,2)$ \\
& Cardiología & $14(15,2)$ \\
Año de residencia & Terapia intensiva adultos & $15(16,3)$ \\
& Primero & $40(43,5)$ \\
& Segundo & $19(20,7)$ \\
Sexo & Tercero & $18(19,6)$ \\
& Cuarto & $15(16,3)$ \\
Nacionalidad & Mujer & $64(69,6)$ \\
Extranjero & Varón & $28(30,4)$ \\
Convivencia & Argentino & $80(87)$ \\
& 12 & $(13)$ \\
& Vive solo & $33(35,9)$ \\
& Vive acompañado & $59(64,1)$ \\
\hline
\end{tabular}

despersonalización (F: 3,49; $p=0,011$ ). No hubo diferencias en el análisis de realización personal.

Según la definición adoptada para BO tomando en cuenta las tres subescalas, 18 encuestados $(19,6 \%)$ presentaban valores compatibles con el síndrome.

Luego de dividir la muestra según la presencia o la ausencia de BO, las dos poblaciones solo mostraron diferencias significativas en la evaluación del clima educacional (Tabla 4).

Para el análisis univariado se dicotomizó CE por su mediana $(109,4)$ y mostró un OR de 6,94 $(1,85-26)$ para la presencia de $\mathrm{BO}$ para medición de CE por debajo de ese valor.

Ninguna de las otras variables mostró asociación en el análisis univariado.

Al evaluar la correlación entre CE (total y cada una de las subescalas del PHEEM) con cada una de las subescalas del MBI, objetivo principal del trabajo, se encontró una correlación negativa entre clima total con agotamiento y despersonalización, y una correlación positiva entre clima total y realización personal.

Al evaluar las subescalas respectivas de ambos instrumentos, se observó una correlación significativa negativa entre autonomía con agotamiento y despersonalización, y positiva con realización, aun con más peso que la correlación con clima total. La subescala de soporte no se correlacionó con BO y la subescala de enseñanza se correlacionó débilmente en forma negativa con despersonalización y positiva con realización (Tabla 5).

Tabla 2. Evaluación del clima educacional. Postgraduate Hospital Educational Environment Measure (PHEEM)

\begin{tabular}{lcccc}
\hline & Media & $\begin{array}{c}\text { Desviación } \\
\text { estándar }\end{array}$ & Mediana & $\begin{array}{c}\text { Rango } \\
\text { IQ 25-75 }\end{array}$ \\
\hline Clima total & 106,8 & 13,98 & 109,4 & $98,5-116$ \\
Autonomía & 36,57 & 5,69 & 37 & $33-40$ \\
Soporte & 30,48 & 2,48 & 31 & $28-34$ \\
Enseñanza & 39,79 & 6,14 & 41 & $35,5-43,5$ \\
\hline
\end{tabular}

TABLA 3. Resultados del Maslach Burnout Inventory

\begin{tabular}{|c|c|c|c|c|}
\hline & Media & $\begin{array}{c}\text { Desviación } \\
\text { estándar }\end{array}$ & Mediana & $\begin{array}{c}\text { Rango } \\
\text { IQ 25-75 }\end{array}$ \\
\hline Agotamiento & 27,14 & 10,37 & 27 & $20,5-34,5$ \\
\hline Despersonalización & 10,73 & 6,51 & 10 & $5-16$ \\
\hline Realización & 37,01 & 4,82 & 38 & $34-40,5$ \\
\hline
\end{tabular}


TABLA 4. Población según la presencia de burnout $(B O)^{*}$

\begin{tabular}{|c|c|c|c|}
\hline & $\begin{array}{c}\text { BO Sí= } 18 \\
(19,6 \%)\end{array}$ & BO No= $74(80,4 \%)$ & $\begin{array}{c}\text { Significación }^{* *} \\
p<0,05\end{array}$ \\
\hline \multicolumn{4}{|l|}{ Especialidad } \\
\hline 1 & 4 & 25 & $p=0,143$ \\
\hline 2 & 5 & 15 & \\
\hline 3 & 1 & 13 & \\
\hline 4 & 6 & 8 & \\
\hline 5 & 2 & 13 & \\
\hline \multicolumn{4}{|l|}{ Año } \\
\hline 1 & 9 & 31 & $p=0,526$ \\
\hline 2 & 5 & 14 & \\
\hline 3 & 3 & 15 & \\
\hline 4 & 1 & 14 & \\
\hline \multicolumn{4}{|l|}{ Sexo } \\
\hline Mujer & 13 & 51 & $p=0,514$ \\
\hline Varón & 5 & 23 & \\
\hline \multicolumn{4}{|l|}{ Nacionalidad } \\
\hline Argentino & 15 & 65 & $p=0,429$ \\
\hline Extranjero & 3 & 9 & \\
\hline \multicolumn{4}{|l|}{ Convivencia } \\
\hline Solo & 7 & 26 & $p=0,484$ \\
\hline Acompañado & 11 & 48 & \\
\hline \multirow[t]{2}{*}{ Clima *** } & 98,7 & 108,8 & $p=0,0056$ \\
\hline & $(92,5-104,9)$ & $(105,6-111,9)$ & \\
\hline
\end{tabular}

* Burnout Sí= agotamiento $>26$ y despersonalización $>9$ y realización $<34$.

** Variables categóricas: $\chi^{2}$, variables continuas: prueba de la $t$.

*** Clima: media (intervalo de confianza 95\%).

TABLA 5. Correlación entre clima total y subescalas del PHEEM (autonomía, soporte y enseñanza), con las tres subescalas del Maslach Burnout Inventory (agotamiento, depersonalización y realización)

\begin{tabular}{lcccc}
\hline & Clima total & Autonomía & Soporte & Enseñanza \\
\hline Agotamiento & $-\mathbf{0 , 2 4 3} \mathrm{p}=\mathbf{0 , 0 1 9 6}$ & $-\mathbf{0 , 5 7 8 6} \mathrm{p}=\mathbf{0 , 0 0 0}$ & $0,0014 \mathrm{p}=0,989$ & $-0,0821 \mathrm{p}=0,4366$ \\
Despersonalización & $-\mathbf{0 , 3 5 3 5} \mathrm{p}=\mathbf{0 , 0 0 0 5}$ & $-\mathbf{0 , 4 0 5 1} \mathrm{p}=\mathbf{0 , 0 0 0 1}$ & $0,0136 \mathrm{p}=0,898$ & $-\mathbf{0 , 2 6 8 2} \mathrm{p}=\mathbf{0 , 0 0 9 8}$ \\
Realización & $\mathbf{0 , 4 4 1} \mathrm{p}=\mathbf{0 , 0 0 0 0}$ & $\mathbf{0 , 4 5 9 3} \mathrm{p}=\mathbf{0 , 0 0 0}$ & $0,1203 \mathrm{p}=0,2532$ & $\mathbf{0 , 3 2 0 4} \mathrm{p}=\mathbf{0 , 0 0 1 8}$ \\
\hline
\end{tabular}

Se destacan en negrita las correlaciones que muestran significación estadística.

La utilización del PHEEM no presentó dificultades en la comprensión de los interrogantes. Se obtuvo un $100 \%$ de encuestas completadas, con la totalidad de los ítems respondidos. Mostró una adecuada confiabilidad con un coeficiente alfa de Chronbach de 0,878.

\section{DISCUSIÓN}

El mejoramiento de la calidad de los programas de formación de profesionales médicos es un desafío permanente para el cual se han utilizado diferentes enfoques.

La evaluación del CE -entendiendo que este constructo refleja la interrelación entre el profesional en etapa de entrenamiento y los diferentes actores del equipo de salud, como también las características institucionales que definen las "culturas" específicas de cada lugares una herramienta eficaz para evaluar un programa.

El CE es un constructo abstracto que se puede medir en forma indirecta a través de instrumentos específicos. Así, Roff y cols. ${ }^{13}$ crearon el Postgraduate Hospital Educational Environment Measure (PHEEM) para evaluar una instancia similar a nuestro internado rotatorio. Este ha sido 
validado al portugués ${ }^{14} \mathrm{y}$ al español, ${ }^{15} \mathrm{y}$ se realizó una evaluación multicéntrica que lo mostró como un instrumento multidimensional y altamente confiable. ${ }^{16}$

En los últimos tiempos, ha ganado atención la presencia del síndrome de BO en la profesión médica, con particular interés en la evaluación de los residentes. Niku, ${ }^{9}$ en su revisión de la literatura de los trabajos de $\mathrm{BO}$ en la residencia, identificó 15 trabajos con distintas limitaciones metodológicas. La prevalencia promedio de $\mathrm{BO}$ informada en diferentes escenarios supera el 50\% de los residentes evaluados. Distintos trabajos han relacionado el $\mathrm{BO}$ con las condiciones de trabajo de los médicos y los enfermeros. ${ }^{17,18}$

Se ha intentado relacionar las características personales y la aparición de BO, pero no se llegó a conclusiones definitivas. ${ }^{19}$

Desde otro enfoque, $\mathrm{McCray}^{7}$ realizó una revisión sistemática de trabajos que evaluaron las intervenciones que impactaban sobre la incidencia de $\mathrm{BO}$ en los residentes. De su revisión se destaca que la prevalencia de BO osciló entre el $47 \%$ y el $76 \%$, sin diferencias claras entre las especialidades (clínicas y quirúrgicas).

Dyrbye y cols. ${ }^{8}$ llevaron adelante un estudio multicéntrico en cinco escuelas de medicina, evaluando el CE y la aparición de $\mathrm{BO}$ en estudiantes de medicina; concluyeron que existía una asociación tanto en el análisis univariado (OR 2,07; IC 1,85 a 2,31) como en el multivariado (OR 1,78 ; IC 1,47 a 2,15). Brazeau y cols., ${ }^{20}$ midieron el BO utilizando también el MBI, y confirmaron la hipótesis de que se correlaciona en forma inversa con las mediciones de empatía y profesionalismo.

En su revisión de la bibliografía, William y cols. ${ }^{21}$ encontraron una prevalencia de entre $27 \%$ y $75 \%$ de $\mathrm{BO}$ en los residentes, con diferencias relacionadas con la especialidad. Todos estos informes previos de la literatura muestran datos de la presencia de $\mathrm{BO}$ superiores a los detectados en nuestra muestra $(19,6 \%)$, aunque se debe tener en cuenta que, en nuestro caso, solo se evaluaron especialidades clínicas (pediatría, clínica, medicina familiar, cardiología y terapia intensiva) y, además, se utilizó una definición muy estricta de $\mathrm{BO}$, en comparación con los otros estudios.

De nuestro análisis se desprenden diferencias significativas entre las especialidades, tanto en la evaluación del CE como en la presencia de $\mathrm{BO}$, lo cual aporta validez a la capacidad de los instrumentos utilizados y en su poder para detectar diferencias en las variables analizadas en diversos contextos.
Respondiendo a la hipótesis del trabajo, cuando se evaluó la correlación entre CE (PHEEM) y BO (MBI), se observó una correlación negativa entre clima y agotamiento y despersonalización (peor clima se correlaciona con mayores puntajes de $\mathrm{BO}$ en las respectivas subescalas del MBI), y una correlación positiva entre clima y sensación de logros obtenidos (mejor clima, mayor sensación de realización personal).

Más interesante aún es haber encontrado que la subescala de autonomía del PHEEM tuvo una correlación de mayor peso, e igual sentido, con los tres dominios del MBI (negativa con agotamiento y despersonalización, y positiva con realización).

Al analizar cuáles eran los ítems de la subescala de autonomía del PHEEM, se vio que corresponden a los que indagan sobre conductas de tipo anticipatorio (información previa de contenidos, carga horaria, tareas adecuadas a la etapa de formación, posibilidad de adquirir habilidades) y aquellos que evalúan la pertenencia al grupo y su funcionamiento (sentirse parte de un equipo, vivir una atmósfera de mutuo respeto en el grupo). Estos hallazgos coinciden con los factores relacionados con BO informados por Eckleberry y cols..$^{22}$ en su estudio realizado con residentes. Otros autores también encontraron una relación entre la autonomía y el $\mathrm{BO},{ }^{23}$ destacándose que esta relación se describió además en profesiones de servicio no médicas. Al respecto, Boada y cols., encontraron una correlación positiva ( $\mathrm{r} 0,344)$ entre autonomía y realización personal en un grupo de asistentes sociales, administrativos, profesores y policías. ${ }^{24}$

Este hallazgo puede ser un punto de partida importante al planear cambios curriculares y reformas estructurales de carácter preventivo, que parecen ser más eficaces que las estrategias de entrenamiento intensivo en el manejo del estrés. ${ }^{25}$

Este enfoque, además, puede ser superador de las estrategias aisladas para prevenir el $\mathrm{BO}$. No se encontró disminución del BO, luego de la disminución aislada de la carga horaria. ${ }^{26}$ Una limitación del estudio fue el hallazgo de solo 18 residentes que cumplían la definición de $\mathrm{BO}$ en los 92 residentes encuestados. Esto no permitió confirmar diferencias significativas de la presencia de $\mathrm{BO}$, por ejemplo, entre especialidades o años de residencia, a pesar de las tendencias observadas en la distribución de frecuencias. Por otro lado, cuando se hizo el análisis de regresión con clima dicotomizado como variable independiente asociada a $\mathrm{BO}$, la escasa cantidad de eventos (residentes con $\mathrm{BO}$ ) no 
permitió ingresar todas las variables demográficas al modelo. Por la misma causa, si bien se encontró un OR de casi 7 entre clima por debajo del valor de su mediana dicotomizado $(109,4)$ y BO (lo que demuestra una buena asociación), el relativo escaso número de residentes con $\mathrm{BO}$ se tradujo en una dispersión importante (IC 95\% 1,85 a 26).

Con respecto a la modalidad de aplicación, aunque se considera que no se incurrió en un sesgo de selección -ya que si bien los residentes eran encuestados sobre su evaluación del programa en el marco de la institución, se mantuvieron estrictas normas de anonimato y confidencialidad de los datos- debe tenerse en cuenta que algunas de las especialidades incorporadas al estudio no aportaron la totalidad de sus residentes (clínica médica y cardiología). Una evaluación con una muestra mayor y que incorporara otras especialidades (quirúrgicas) podría agregar peso a la correlación encontrada entre el CE y el BO.

\section{CONCLUSIONES}

Se encontró una correlación estadísticamente significativa, de sentido negativo, entre clima educacional con agotamiento y despersonalización, y una correlación estadísticamente significativa y positiva entre $\mathrm{CE}$ y realización personal. A mejor puntaje de CE, menos agotamiento y despersonalización y mejor sentimiento de realización personal.

Una mayor correlación, de igual sentido, se observó entre las subescalas de autonomía del PHEEM y las tres subescalas del MBI.

\section{BIBLIOGRAFÍA}

1. Genn JM, Harden RM. What is Medical Education Here Really Like? Suggestions for action research studies of climates of medical education environments. Med Teach 1986;8(2):111.

2. Genn JM. AMEE Medical Education Guide No. 23 (Part 1): Curriculum, environment, climate, quality and change in medical education-a unifying perspective. Med Teach 2001;23(4):337-44.

3. Genn JM. AMEE Medical Education Guide No. 23 (Part 2): Curriculum, environment, climate, quality and change in medical education-a unifying perspective. Med Teach 2001;23(5):445-54.

4. Bris MM. Clima de trabajo y organizaciones que aprenden. Educar 2000;27:103-117.

5. Mc Aleer. A practical guide for Medical Teacher. Edited by Dent J and Harden R. 3 th.Ed. Churchill Livingstone; 2009.

6. Hutchinson L. Educational environment. BMJ 2003;326:810-2.
7. McCray L, Cronholm P, Bogner H, Gallo J, Neill R. Resident Physician Burnout: Is There Hope? Fam Med 2008;40(9):626-32.

8. Dyrbye L, Thomas M, Shanafelt T. Systematic Review of Depression, Anxiety and Other Indicators of Psychological Distress Among U.S. and Canadian MedicalStudents. Acad Med 2006;81:354-73.

9. Niku Thomas Resident Burnout. JAMA 2004;292(23):2880-9.

10. Eckleberry J, Lick D, Boura J, Hunt R, et al. An Exploratory Study of Resident Burnout and Wellness. Acad Med 2009;84(2):269-77.

11. Maslach C, Jackson SE, Leiter MP. Maslach Burnout Inventory Manual. $3^{\circ}$ ed; CPP Inc, 1996.

12. Rafferty JP, LemkauJP, Purdy RR, RudisillJR. Validity of the Maslach Burnout Inventory for family practice physicians. J Clin Psychol 1986;42:488-92.

13. RoffS., McAleerS, Skinner A. Development and Validation of an instrument to measure postgraduate clinical learning and teaching educational environment for hospitalbased junior doctors in the UK. Med Teach 2005;27(4):326-31.

14. Edson Vieira J. The postgraduate Hospital Educational Environment Measure (PHEEM) questionary identifies quality of instruction as a key factor predicting academic achievement. Clinics 2008;63(6):741-6.

15. Riquelme A, Herrera C, Aranis C, Oporto J, Padilla O. Psychometric analyses and internal consistency of the PHEEM questionnaire to measure the clinical learning environment in the clerkship of a Medical School in Chile. Med Teach 2009;31:6,e:221-5.

16. Wall D, Clapham M, Riquelme A, Vieira J, Cartmill R. Is PHEEM a multidimensional instrument? An international perspective. Med Teach 2009;31:e521-7.

17. Linzer M, Visser M, Oort F. Predicting and Preventing physician burnout: results from the United States and the Netherlands. Am J Med 2001;111:170-5.

18. Aiken L, Clarke S, Sloane D, Sochalski J, Silver J. Hospital nurse staffing and patient mortality, nurse burnout and job dissatisfaction. JAMA 1997;288:1987-93.

19. Nyssen A, Hansez I, Baele P, Lamy M, De Keyser V Occupational stress and burnout in anaesthesia. BrJAnaesth 2003;90:333-7.

20. Brazeau C, Schroeder R, Sue R, Boyd L. Relationship Between Medical Student Burnout, Emphaty, and professionalism Climate. Acad Med 2010;85(10)s33-6.

21. William W, Lederer S, Mandili C, Nikravesh R, et al. Burnout During Residency training. J Grad Med Educ 2009. Págs.236-42.

22. Eckleberry J, Lick D, Boura J, Hunt R, et al. An Exploratory Study of Resident burnout and Wellness. Acade Medic 2009;2:269-77.

23. Ortega Ruiz C, López Ríos F. El burnout o síndrome de estar quemado en los profesionales sanitarios: revisión y perspectivas. Int J Clin Health Psychol 2004;4(1):137-60.

24. Boada J, de Diego Vallejo R, Agulló Tomás E. El Burnout y las manifestaciones pscosomáticas como consecuentes del clima organizacional y de la motivación laboral. Psicothema 2004;16(1):125-31.

25. Dyrbye L, Thomas M, Harper W, Massie SF, et al. The learning environment and medical student burnout: a multicentre study. Med Educ 2009;43:274-82.

26. Gelfand DV, Podnos YD, Carmichael JC, Saltzman DJ, et al. Effect of the 80-hour workweek on resident burnout. Arch Surg 2004;139 (9):933-8. 


\section{Anexo electrónico 1. Cuestionario PHEEM}

\begin{tabular}{|c|c|c|c|c|c|}
\hline & $\begin{array}{c}\text { Fuerte } \\
\text { desacuerdo }\end{array}$ & Desacuerdo & Incierto & Acuerdo & $\begin{array}{l}\text { Fuerte } \\
\text { acuerdo }\end{array}$ \\
\hline \multicolumn{6}{|l|}{$\begin{array}{l}\text { Tengo un programa que provee información } \\
\text { acerca de las horas de actividad clínica. }\end{array}$} \\
\hline \multicolumn{6}{|l|}{$\begin{array}{l}\text { Mis profesores clínicos establecen expectativas } \\
\text { claras. }\end{array}$} \\
\hline \multicolumn{6}{|l|}{$\begin{array}{l}\text { Tengo tiempo educacional protegido en esta } \\
\text { rotación clínica. }\end{array}$} \\
\hline \multicolumn{6}{|l|}{ Tuve un programa de introducción informativo. } \\
\hline \multicolumn{6}{|l|}{$\begin{array}{l}\text { Tengo el nivel apropiado de responsabilidad en } \\
\text { esta rotación clínica. }\end{array}$} \\
\hline \multicolumn{6}{|l|}{$\begin{array}{l}\text { Tengo buena supervisión clínica en todo } \\
\text { momento. }\end{array}$} \\
\hline \multicolumn{6}{|l|}{ Hay racismo en esta rotación clínica. } \\
\hline \multicolumn{6}{|l|}{$\begin{array}{l}\text { Tengo que realizar tareas inapropiadas a mi etapa } \\
\text { de formación. }\end{array}$} \\
\hline \multicolumn{6}{|l|}{$\begin{array}{l}\text { Hay un manual informativo de la rotación clínica } \\
\text { para los residentes. }\end{array}$} \\
\hline \multicolumn{6}{|l|}{$\begin{array}{l}\text { Mis profesores clínicos tienen buenas destrezas } \\
\text { comunicacionales. }\end{array}$} \\
\hline \multicolumn{6}{|l|}{$\begin{array}{l}\text { Soy localizado ("beepeado" o ubicado en mi } \\
\text { teléfono celular) de forma inapropiada. }\end{array}$} \\
\hline \multicolumn{6}{|l|}{$\begin{array}{l}\text { Existen las facilidades para participar en otras } \\
\text { actividades docentes sin interferir con clases o } \\
\text { pruebas de evaluación de otros cursos. }\end{array}$} \\
\hline \multicolumn{6}{|l|}{$\begin{array}{l}\text { Hay discriminación por sexo en esta rotación } \\
\text { clínica }\end{array}$} \\
\hline \multicolumn{6}{|l|}{$\begin{array}{l}\text { Tengo guías claras acerca de mis actividades } \\
\text { clínicas en esta rotación. }\end{array}$} \\
\hline \multicolumn{6}{|l|}{ Mis profesores clínicos son entusiastas. } \\
\hline \multicolumn{6}{|l|}{$\begin{array}{l}\text { Tengo buena colaboración con otros residentes de } \\
\text { mi nivel. }\end{array}$} \\
\hline \multicolumn{6}{|l|}{ Mi horario de actividades clínicas es adecuado. } \\
\hline \multicolumn{6}{|l|}{$\begin{array}{l}\text { Tengo la oportunidad de ofrecer continuidad en } \\
\text { el cuidado de los pacientes. }\end{array}$} \\
\hline \multicolumn{6}{|l|}{ Tengo acceso adecuado a consejería. } \\
\hline \multicolumn{6}{|l|}{$\begin{array}{l}\text { Esta rotación clínica (hospital/consultorio) } \\
\text { tiene una buena calidad de espacios físicos para } \\
\text { desarrollar actividades docentes. }\end{array}$} \\
\hline \multicolumn{6}{|l|}{$\begin{array}{l}\text { Hay acceso a un programa educacional relevante } \\
\text { con objetivos explícitos para mis necesidades. }\end{array}$} \\
\hline \multicolumn{6}{|l|}{$\begin{array}{l}\text { Recibo retroalimentación (feedback) regularmente } \\
\text { de parte de los docentes. }\end{array}$} \\
\hline \multicolumn{6}{|l|}{ Mis profesores clínicos son bien organizados. } \\
\hline \multicolumn{6}{|l|}{$\begin{array}{l}\text { Me siento físicamente seguro en el ambiente } \\
\text { clínico (hospitalario / ambulatorio). }\end{array}$} \\
\hline \multicolumn{6}{|l|}{$\begin{array}{l}\text { Hay una cultura de no culpar en esta rotación } \\
\text { clínica. }\end{array}$} \\
\hline $\begin{array}{l}\text { Hay facilidades adecuadas para obtener comida y } \\
\text { bebidas (cafetería). }\end{array}$ & & & & & \\
\hline $\begin{array}{l}\text { Tengo suficientes oportunidades de aprendizaje } \\
\text { clínico para mis necesidades. }\end{array}$ & & & & & \\
\hline
\end{tabular}




\begin{tabular}{|c|c|c|c|c|c|}
\hline & $\begin{array}{c}\text { Fuerte } \\
\text { desacuerdo }\end{array}$ & Desacuerdo & Incierto & Acuerdo & $\begin{array}{c}\text { Fuerte } \\
\text { acuerdo }\end{array}$ \\
\hline \multicolumn{6}{|l|}{$\begin{array}{l}\text { Mis profesores clínicos tienen buenas destrezas } \\
\text { clínicas. }\end{array}$} \\
\hline \multicolumn{6}{|l|}{ Aquí me siento parte de un equipo de trabajo. } \\
\hline \multicolumn{6}{|l|}{$\begin{array}{l}\text { Tengo oportunidades de adquirir los } \\
\text { procedimientos prácticos apropiados para mi } \\
\text { nivel. }\end{array}$} \\
\hline \multicolumn{6}{|l|}{ Mis profesores clínicos son asequibles. } \\
\hline \multicolumn{6}{|l|}{$\begin{array}{l}\text { Mi carga de trabajo en esta rotación clínica es } \\
\text { adecuada. }\end{array}$} \\
\hline \multicolumn{6}{|l|}{$\begin{array}{l}\text { Los docentes utilizan las oportunidades de } \\
\text { aprendizaje en forma efectiva. }\end{array}$} \\
\hline \multicolumn{6}{|l|}{$\begin{array}{l}\text { El entrenamiento en esta rotación clínica me hace } \\
\text { sentir preparado para ser médico. }\end{array}$} \\
\hline \multicolumn{6}{|l|}{$\begin{array}{l}\text { Mis profesores clínicos tienen buenas destrezas } \\
\text { como mentores. }\end{array}$} \\
\hline \multicolumn{6}{|l|}{$\begin{array}{l}\text { Tengo tiempo de esparcimiento fuera de las } \\
\text { actividades de esta rotación clínica. }\end{array}$} \\
\hline \multicolumn{6}{|l|}{$\begin{array}{l}\text { Mis profesores clínicos me fomentan el } \\
\text { aprendizaje independiente. }\end{array}$} \\
\hline \multicolumn{6}{|l|}{$\begin{array}{l}\text { Existen buenas oportunidades de consejería para } \\
\text { alumnos que fallan en esta rotación clínica. }\end{array}$} \\
\hline \multicolumn{6}{|l|}{$\begin{array}{l}\text { Los profesores clínicos me proveen buena } \\
\text { retroalimentación (feedback) respecto de mis } \\
\text { fortalezas y debilidades. }\end{array}$} \\
\hline $\begin{array}{l}\text { Mis tutores clínicos promueven una atmósfera de } \\
\text { mutuo respeto. }\end{array}$ & & & & & \\
\hline
\end{tabular}

\section{INTERPRETACIÓN DE LOS PUNTAJES DEL PHEEM}

La evaluación del clima total tiene la siguiente interpretación:

0 a 40: muy pobre

41 a 80: lleno de problemas

81 a 120: más positivo que negativo

121 a 160: excelente

La subescala de autonomía tiene la siguiente interpretación:

Ítems 1, 4, 5, 8, 9, 11, 14, 17, 18, 29, 30, 32, 34, 40

0 a 14 : muy pobre

15 a 28: negativa

29 a 42: positiva

43 a 56: excelente

La subescala de soporte tiene la siguiente interpretación Ítems: 7, 13, 19, 20, 24, 25, 26, 35, 38

0 a 11: no existe

12 a 22: no es un lugar placentero

23 a 33: más pros que contras

34 a 44: ambiente con buen soporte

La subescala de enseñanza tiene la siguiente interpretación:

Ítems: 2, 3, 6, 10, 12, 15, 21, 22, 23, 27, 28, 31, 33, 37, 39

0 a 15 : muy pobre

16 a 30: necesita revisión

31 a 45: en buena dirección

46 a 60: maestros modelos

Los ítems 7, 8, 11 y 13 puntúan en forma inversa. 


\section{Anexo electrónico 2. Maslach Burnout Inventory (MBI)}

\begin{tabular}{|c|c|c|c|c|c|c|c|c|}
\hline & & Nunca & \begin{tabular}{|c|} 
Alguna \\
vez al \\
año o menos \\
\end{tabular} & $\begin{array}{c}\begin{array}{c}\text { Una vez } \\
\text { al } \\
\text { mes o menos }\end{array} \\
\end{array}$ & $\begin{array}{l}\text { Algunas } \\
\text { veces } \\
\text { al mes } \\
\end{array}$ & $\begin{array}{c}\text { Una } \\
\text { vez a la } \\
\text { semana }\end{array}$ & \begin{tabular}{|c|} 
Varias \\
veces a la \\
semana \\
\end{tabular} & Diariamente \\
\hline & & 0 & 1 & 2 & 3 & 4 & 5 & 6 \\
\hline 1 & $\begin{array}{l}\text { Debido a mi trabajo me siento } \\
\text { emocionalmente agotado. }\end{array}$ & & & & & & & \\
\hline 2 & $\begin{array}{l}\text { Al final de la jornada me siento } \\
\text { agotado. }\end{array}$ & & & & & & & \\
\hline 3 & $\begin{array}{l}\text { Me encuentro cansado cuando me } \\
\text { levanto por las mañanas y tengo que } \\
\text { enfrentarme a otro día de trabajo. }\end{array}$ & & & & & & & \\
\hline 4 & $\begin{array}{l}\text { Puedo entender con facilidad lo que } \\
\text { piensan mis pacientes. }\end{array}$ & & & & & & & \\
\hline 5 & $\begin{array}{l}\text { Creo que trato a algunos pacientes } \\
\text { como si fueran objetos. }\end{array}$ & & & & & & & \\
\hline 6 & $\begin{array}{l}\text { Trabajar con pacientes todos los días } \\
\text { es una tensión para mí. }\end{array}$ & & & & & & & \\
\hline 7 & $\begin{array}{l}\text { Me enfrento muy bien con los } \\
\text { problemas que me presentan mis } \\
\text { pacientes. }\end{array}$ & & & & & & & \\
\hline 8 & Me siento "quemado" por el trabajo. & & & & & & & \\
\hline 9 & $\begin{array}{l}\text { Siento que mediante mi trabajo } \\
\text { estoy influyendo positivamente en } \\
\text { la vida de otros. }\end{array}$ & & & & & & & \\
\hline 10 & $\begin{array}{l}\text { Creo que tengo un comportamiento } \\
\text { más insensible con la gente desde } \\
\text { que hago este trabajo. }\end{array}$ & & & & & & & \\
\hline 11 & $\begin{array}{l}\text { Me preocupa que este trabajo me } \\
\text { esté endureciendo emocionalmente. }\end{array}$ & & & & & & & \\
\hline 12 & Me encuentro con mucha vitalidad. & & & & & & & \\
\hline 13 & Me siento frustrado por mi trabajo. & & & & & & & \\
\hline 14 & $\begin{array}{l}\text { Siento que estoy haciendo un trabajo } \\
\text { demasiado duro. }\end{array}$ & & & & & & & \\
\hline 15 & $\begin{array}{l}\text { Realmente no me importa lo que les } \\
\text { ocurrirá a algunos de los pacientes a } \\
\text { los que tengo que atender. }\end{array}$ & & & & & & & \\
\hline 16 & $\begin{array}{l}\text { Trabajar en contacto directo con } \\
\text { los pacientes me produce bastante } \\
\text { estrés. }\end{array}$ & & & & & & & \\
\hline 17 & $\begin{array}{l}\text { Tengo facilidad para crear una } \\
\text { atmósfera relajada a mis pacientes. }\end{array}$ & & & & & & & \\
\hline$\overline{18}$ & $\begin{array}{l}\text { Me encuentro animado después de } \\
\text { trabajar junto con los pacientes. }\end{array}$ & & & & & & & \\
\hline 19 & $\begin{array}{l}\text { He realizado muchas cosas que } \\
\text { merecen la pena en este trabajo. }\end{array}$ & & & & & & & \\
\hline 20 & $\begin{array}{l}\text { En el trabajo siento que estoy al } \\
\text { límite de mis posibilidades. }\end{array}$ & & & & & & & \\
\hline 21 & $\begin{array}{l}\text { Siento que sé tratar de forma } \\
\text { adecuada los problemas } \\
\text { emocionales en el trabajo. }\end{array}$ & & & & & & & \\
\hline 22 & $\begin{array}{l}\text { Siento que los pacientes me culpan } \\
\text { de algunos de sus problemas. }\end{array}$ & & & & & & & \\
\hline
\end{tabular}


Subescala de agotamiento emocional. Consta de 9 preguntas. Valora la vivencia de estar exhausto emocionalmente por las demandas del trabajo. Puntaje máximo 54. La conforman los ítems 1, 2, 3, 6, 8, 13, 14, 16 y 20. Su puntaje es directamente proporcional a la intensidad del síndrome. El puntaje máximo es de 54; cuanto mayor es el puntaje en esta subescala, mayor es el agotamiento emocional y el nivel de $\mathrm{BO}$ experimentado por el sujeto.

Subescala de despersonalización. Está formada por 5 ítems: 5, 10, 11, 15 y 22. Valora el grado en que cada uno reconoce actitudes de frialdad y distanciamiento. El puntaje máximo es de 30; cuanto mayor es el puntaje en esta subescala, mayor es la despersonalización y el nivel de BO experimentado por el sujeto.

Subescala de realización personal. Se compone de 8 ítems. Evalúa los sentimientos de autoeficacia y realización personal en el trabajo. La realización personal está conformada por los ítems 4, 7, 9, 12, 17, 18, 19 y 21. El puntaje máximo es de 48; cuanto mayor es el puntaje en esta subescala, mayor es la realización personal porque en este caso el puntaje es inversamente proporcional al grado de BO. Es decir, a menor puntaje de realización o logro personal más afectado está el sujeto. 